\title{
Circulación inducida por el viento en Bahía de Antofagasta, norte de Chile $\left(23^{\circ} \mathrm{S}\right)$
}

\author{
Wind-driven circulation in Bahía de Antofagasta, northern Chile (23 S) \\ Ruben Escribano $^{1}$ y Pamela Hidalgo ${ }^{2}$ \\ ${ }^{1}$ Instituto de Investigaciones Oceanológicas, Universidad de Antofagasta. Casilla 170, Antofagasta \\ ${ }^{2}$ Departamento de Oceanografía, Universidad de Concepción. Casilla 160 C, Concepción \\ rescribano@uantof.cl
}

\begin{abstract}
Resumen.- Se estudió la circulación de la bahía de Antofagasta $\left(23^{\circ} \mathrm{S}\right)$ sobre las base de mediciones de series de tiempo de corrientes en verano e invierno de 1999, de un crucero oceanográfico realizado en agosto de 1999 y una serie de 9 meses de perfiles de corrientes a intervalos de 15 días. La información de corrientes sumada a datos de temperatura, salinidad y oxígeno, e información de vientos, permitió analizar la variabilidad temporal y espacial de la circulación en la bahía. La bahía de Antofagasta está sujeta a una intensa variación de los vientos, a la cual las corrientes se manifiestan muy asociadas y en desfases de tiempo de 1 hasta 5 días. El ciclo que presenta mayor contribución a la varianza total de la corriente es en la escala diaria. La bahía presenta circulación en una capa, con velocidades medias de $30 \mathrm{~cm} \mathrm{~s}^{-1}$. El campo de corrientes sugiere que la bahía se llena a través del sector norte y su vaciamiento ocurre en el extremo sur. La presencia de un foco de surgencia en el sector sur externo a la bahía ejerce una fuerte influencia en la circulación, a través del influjo de aguas de surgencia desde el sur y dando orígen a una zona frontal que modula el llenado y vaciado de la bahía. El estudio presenta un modelo conceptual de circulación sujeta a la variabilidad en la intensidad de surgencia. El modelo predice que bajo condiciones de relajación de surgencia y el establecimiento de zona frontal en la boca sur la circulación sería dominada por un giro ciclónico al interior de la bahía, y frente a pulsos de intensificación de la surgencia se produciría el llenado. Se discuten las implicancias de los resultados en el contexto de la capacidad de retención y renovación de aguas de la bahía.

Palabras claves: Circulación, surgencia, corrientes, variabilidad.
\end{abstract}

\begin{abstract}
Circulation of Bahía de Antofagasta (23 S) was studied based on time series measurements of currents in summer and winter 1999, an oceanographic survey performed in August 1999, and a 9-months series of current profiling at $15 \mathrm{~d}$ time intervals. Current data, along with information on temperature, salinity, oxygen, and wind data, allowed the analysis of temporal and spatial variation of circulation within the bay. Bahía de Antofagasta is subject to intense variability of winds, to which currents seemed strongly associated, in time lags from 1 to $5 \mathrm{~d}$. The cycle that most contributed to total variance of currents is in time scales of days. The bay showed a one-layer circulation at mean velocities of about 30 $\mathrm{cm} \mathrm{s}^{-1}$. The current field suggested that inflow to the bay takes place at the northern end, and the outflow through the southern opening. Presence of an upwelling focus at the external area in the south exerts a strong influence on the circulation, by causing upwelled water to enter the bay from the south, and giving rise to a front that regulates water inflow/outflow. We present a conceptual model of circulation subject to variability in intensity of upwelling. The model predicts that under upwelling-relaxation conditions and establishment of the front in the southern opening, circulation would be dominated by a cyclonic gyre inside the bay, and that intense pulses of upwelling would induce water inflow. This work discusses the implications of our results in the context of water retention and renewal capacities of the water body of the bay.
\end{abstract}

Keywords: Circulation, upwelling, currents, variability.

\section{Introducción}

El área norte del Sistema de la Corriente de Humboldt (SCH) presenta una costa predominantemente rectilínea, sin mayores indentaciones y alineada en el sentido surnorte. La plataforma continental es estrecha $y$ en algunos sectores virtualmente inexistente. Entonces, la mayoría de la productividad biológica ocurre en una banda estrecha a lo largo de la costa, en particular en áreas de surgencia muy activa (Fonseca \& Farías 1987). Así la estrecha franja costera constituye una zona clave de producción biológica del $\mathrm{SCH}$, reflejando el ampliamente reconocido rol de los márgenes costeros en la productividad de los océanos (Walsh 1991). En este márgen continental la alta productividad biológica es promovida por la surgencia costera inducida por los 
vientos sur y suroeste (Alheit \& Bernal 1983). La surgencia fertiliza la zona eufótica promoviendo altos niveles de producción nueva (Barber \& Smith 1981). Es necesario hacer notar también que la zona costera es el lugar donde se concentra la explotación de recursos marinos, junto a las actividades propias del uso industrial y urbano del litoral. De tal manera que es ineludible una comprensión cabal de los procesos físicos, químicos y biológicos para desarrollar apropiadas medidas de manejo y protección del ecosistema marino-costero.

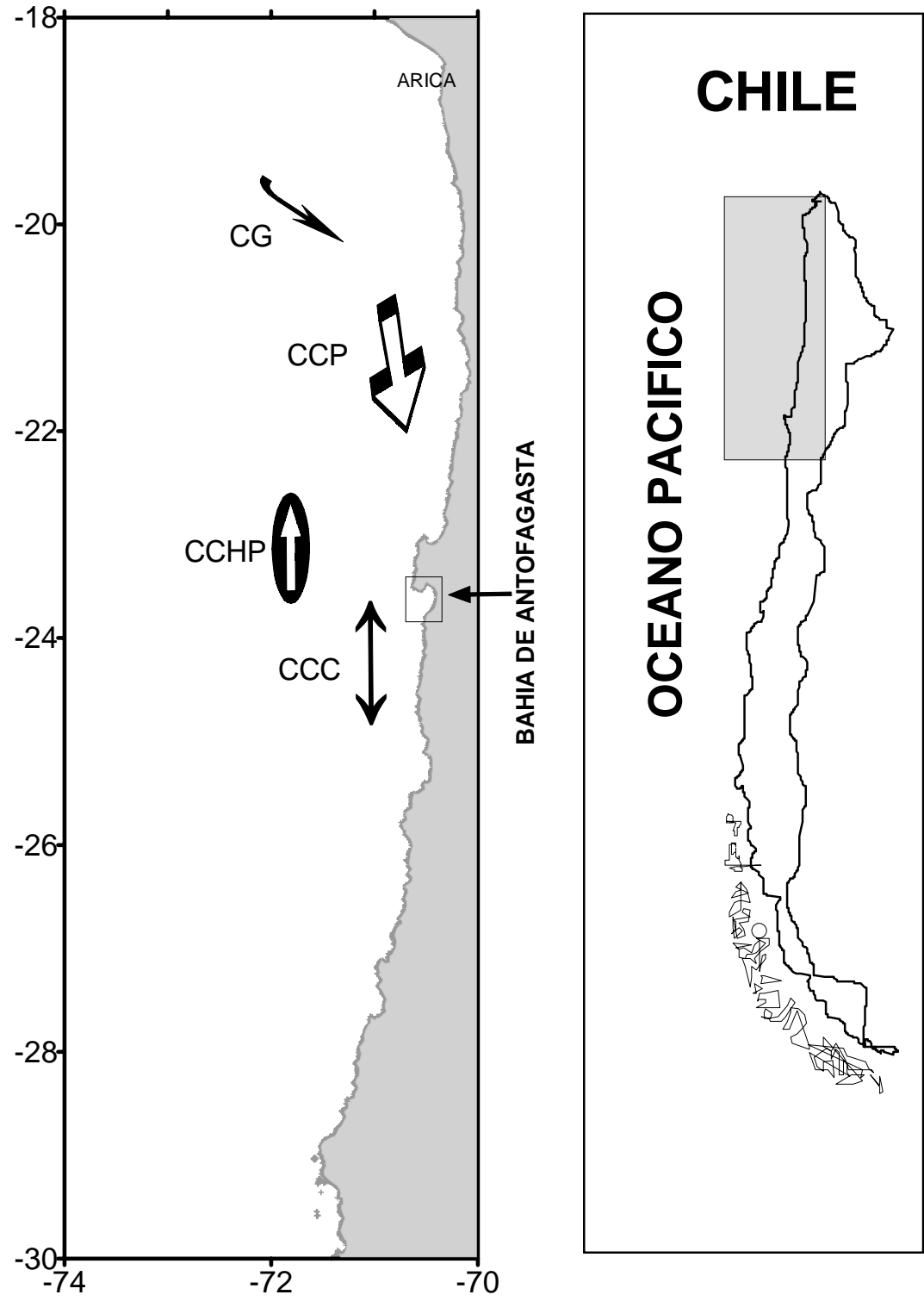

Figura 1

El área norte del Sistema de la Corriente de Humboldt, ilustrando la dinámica de gran escala de corrientes marinas, y la localización de la Bahía de Antofagasta. CG= Corriente subsuperficial de Gunther, CCP= Contracorriente Peruana, CCHP= Corriente Chile-Perú, CCC= Corriente Chilena Costera.

The northern area of the Humboldt Current System and the large scale circulation patterns off Bahía de Antofasta. CG= Subsurface Gunther Current, CCP= Peruvian Countercurrent, CCHP= Chile-Peru Current, CCC= Chilean Coastal Current. 
Strub et al. (1998) resumieron los procesos físicos que gobiernan la circulación de gran escala en el SCH. La Fig.1 ilustra la zona norte del SCH y la localización de bahía de Antofagasta, así como las principales corrientes que interactuan en el norte de Chile en una gran escala espacial. Sin embargo, los estudios de mesoescala, y especialmente los procesos y patrones de circulación que toman lugar en ambientes muy costeros, son muy escasos en la zona norte de Chile. Observaciones en la zona de Antofagasta, a través de imágenes satelitales muestran la presencia aguas frías de surgencia muy localizadas en el borde costero (Fonseca \& Farías 1987, Escribano \& McLaren 1999), posiblemente fertilizando áreas expuestas, así como zonas semicerradas, de las cuales la bahía de Antofagasta, podría directamente ser influenciada y enriquecida (Marin et al. (en prensa), Escribano et al. 2000a). Tal efecto de la surgencia costera sobre la bahía de Antofagasta podría explicar las altas concentraciones del fitoplancton y riqueza de especies que en ella ocurren a través del año (Rodriguez et al. 1996), así como las agregaciones de zooplancton (Escribano \& Hidalgo 2000, Escribano et al. 2000b) que se suceden en períodos primavera-verano, cuando la surgencia se intensifica en la zona (Fonseca \& Farías 1987). Esta persistencia de agregaciones planctónicas al interior de la bahía, sugiere la presencia de procesos físicos que ayudan a la mantención de los organismos. En otras zonas de surgencia se han descrito procesos tales como, presencia de frentes termales que retienen masas de aguas hacia la costa (Graham \& Largier 1997), circulación en doble capa que permiten flujos compensatorios en el plano vertical (Wroblewski 1982, Peterson 1998), y giros inducidos por la morfología costera (Wing et al. 1998, Hutchings et al. 1995). Ninguno de tales procesos ha sido estudiado en bahía de Antofagasta, no obstante estar sujeta a un activo centro de surgencia y así constituir un área clave como zona de retención de poblaciones planctónicas y meroplanctónicas. Es indudable que el conocimiento de la dinámica física de esta bahía posee variados alcances en el ámbito científico y aplicado, no solo por constituir un ambiente clave de organismos marinos, sino que además la bahía de Antofagasta concentra una intensa y creciente actividad industrial y urbana, requiriendo en forma urgente información básica para su óptimo manejo y establecer adecuadas bases para la elaboración de su plano regulador costero.

Para comprender la dinámica de aguas en la bahía de Antofagasta, es necesario en primer lugar considerar los procesos físicos de mayor escala que controlan las masas de agua en la zona. Frente a Antofagasta se desarrolla una activa mezcla de masas de aguas, y cambios en las corrientes como resultado de la interacción de cuatro flujos mayores: la contracorriente superficial Perú-Chile dirigida al Polo, la Corriente Chile-Perú hacia el Ecuador, la corriente subsuperficial de Gunther hacia el Polo que se aproxima hacia la costa en la zona norte de Chile, y la corriente costera chilena la cual es altamente variable en sentido e intensidad (Robles et al. 1976, Fonseca 1989, Silva \& Sievers 1981, Strub et al. 1998). Como resultado la bahía de Antofagasta, debería estar sujeta a un flujo variable surnorte paralelo a la costa, derivado de la corriente costera chilena. Sin embargo, su orientación hacia el sur y su morfología costera, sumada a una forzante de vientos predominantemente sur y suroeste en la zona (Rutllant et al. 1998), sugieren la existencia de un giro en su interior. En tal condición, se puede generar una zona inercial de baja energía que favorecería enormemente la retención de aguas. En este trabajo, sobre la base de mediciones directas de corrientes en series de tiempo y espaciales, se somete a prueba tal hipótesis y se discuten las implicancias de la circulación de la bahía en términos de zona de retención y su capacidad de renovación de aguas.

\section{Materiales y Métodos}

En la bahía de Antofagasta los estudios de medición de corrientes se realizaron en distintos períodos durante 1999 y 2000. La Fig. 2 muestra la localización de estaciones de muestreo para el fondeo de un correntómetro y los puntos de medición de corrientes instantáneas. Durante febrero-marzo de 1999 y julioseptiembre de 1999 se fondeó un correntómetro Aanderaa RCM-8 en el sector Rinconada de la bahía en la isobata de $30 \mathrm{~m}$ (Fig. 2), a una profundidad de $8 \mathrm{~m}$ y configurado para obtener vectores promedios horarios de corrientes. Esta información permitió contar con dos series de tiempo de 40 días y cerca de 90 días para las fechas de verano e invierno, respectivamente. Debido a lecturas erráticas de los últimos días, por efecto de organismos incrustantes en el rotor del equipo, se descontaron mediciones de ambas series. De tal manera, se consideraron 31 días y 75 días para el verano e invierno respectivamente. Para los mismos períodos se utilizó información de 30 días de vientos, obtenida desde la base meteorológica del aeropuerto Cerro Moreno de Antofagasta (Fig. 2), la cual fue constrastada con información de vientos obtenida en Punta Coloso.

Durante el mes de agosto de 1999 se realizó un crucero a bordo de la embarcación L/C PuriHaalar de la Universidad de Antofagasta, que cubrió un total de 14 estaciones en el interior y en la zona externa frente al eje de entrada de la bahía (Fig. 2). Para obtener perfiles verticales de corrientes se utilizó un correntómetro Doppler Aanderaa RCM-9, equipado con sensores calibrados de conductividad, temperatura, oxígeno disuelto y presión. La medición de corrientes se realizó sobre una transecta que une ambas puntas de la bahía 
(Fig. 2), junto a estaciones adicionales de CTD SeaBird SBE-19, desde la superficie hasta un máximo de $500 \mathrm{~m}$ en la zona externa. Las mediciones de corrientes se realizaron en vectores medios replicados (2-3 réplicas) de intervalos de 1 minuto, en 3 capas principales: superficial (0-10 m), media (10-50) y profunda (50- 200 $\mathrm{m})$. Este experimento se realizó por única vez y el período de medición de corrientes instantáneas correspondió a un total de 3.5 horas. Adicionalmente, desde diciembre de 1999 a agosto 2000, en condiciones de calma y marea llenante, cada 15 días se obtuvieron perfiles de corrientes en 2 puntos separados por cerca de $500 \mathrm{~m}$, en el sector La Rinconada (Fig. 2), desde una profundidad de $50 \mathrm{~m}$ y $20 \mathrm{~m}$ respectivamente hasta superficie, con el objeto de estudiar la variabilidad estacional de la capa superficial en un ciclo anual.

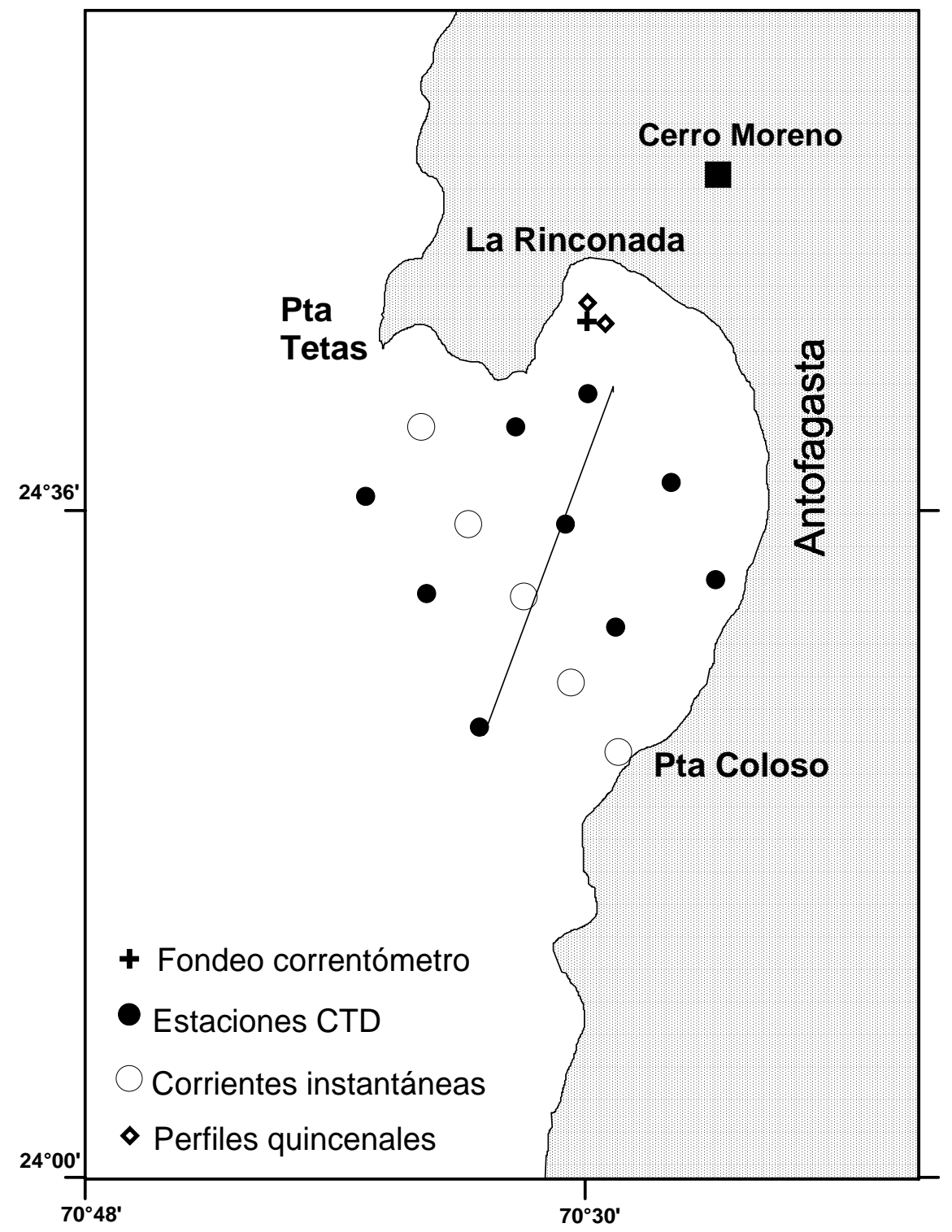

Figura 2

Bahía de Antofagasta mostrando las estaciones de fondeo de correntómetro durante los períodos febrero/marzo y julio/septiembre de 1999, los puntos de medición quincenal de corrientes instantáneas en La Rinconada, y las estaciones oceanográficas y de mediciones directas de corrientes durante el día 16 de agosto de 1999. La línea continua indica el eje de la bahía para las secciones transversales de la Fig.11.

Bahía de Antofagasta illustrating the current meter mooring stations during the periods February/March and July/September 1999, the stations for profiling instantaneous currents (15 d intervals) at La Rinconada, and the oceanographic stations for CTDO and direct measurements of instantaneous currents on August 16, 1999. The continuous line indicates the axis for the oceanographic sections in Fig.11. 
La información se analizó mediante métodos gráficos y de análisis de series de tiempo para estudiar el efecto del viento y cambios en la marea sobre las corrientes, además de filtrar señales de alta frecuencia. Las series de tiempo de corrientes se corrigieron para la variación magnética del área, descomponiéndose en sus ejes norte-sur y este-oeste. La presencia de ciclos alternados de cambios en intensidad y dirección de las corrientes se estudió mediante funciones de autocorrelación y de análisis de Fourier, mientras que las asociaciones se analizaron mediante métodos de correlación cruzada. Para evaluar el efecto de la marea sobre las corrientes, se obtuvo la predicción de altura horaria del mar mediante método descrito por Beer (1989), utilizando como valores de reducción de sonda aquellos reportados por las Tablas de Servicio Hidrográfico de la Armada de Chile (SHOA) del año 1999 para el puerto de Antofagasta. Una vez evaluada la correlación entre la corriente y la marea en las bandas de 24 horas, se procedió a filtrar el efecto mareal, utilizando un filtro pasa-bajo "lowess", con una tensión de 0.05 , lo cual permite remover variaciones en bandas inferiores a 24 horas (Box \& Jenkins 1976). La significancia estadística se obtuvo mediante pruebas paramétricas, sobre variables normalizadas por transformación logarítmica. Las mediciones de corrientes instantáneas se promediaron como vectores para las capas descritas anteriormente y graficaron sus magnitudes y direcciones geográficas. La serie quincenal de corrientes instantáneas en los dos puntos del sector La Rinconada se interpoló mediante interpolación lineal simple sobre la profundidad y el tiempo para construir el contorno de isolíneas. La información oceanográfica complementaria referida a temperatura, salinidad y oxígeno disuelto se utilizó para establecer las condiciones físicas y químicas del cuerpo de agua de la bahía. Finalmente la información integrada se utilizó para proponer un modelo conceptual de circulación en la bahía.

\section{Resultados}

\section{Variabilidad temporal}

Para el período de verano el viento predominante fue sur con una ocurrencia de $66.6 \%$ y una intensidad media de $8.2 \mathrm{~m} \mathrm{~s}^{-1}$, mientras que en invierno la ocurrrencia del viento sur fue de $57.5 \%$ con una velocidad media de 9.3 $\mathrm{m} \mathrm{s}^{-1}$. En ambas estaciones el viento sur se presentó con mayor ocurrencia durante horas de la tarde y las reversiones diarias en su dirección se sucedieron hacia la madrugada con velocidades medias en el rango de 6 a $7 \mathrm{~m} \mathrm{~s}^{-1}$.

Sobre la base del análisis de las series de corrientes horarias de verano e invierno, en el sector La Rinconada la corriente fue altamente variable en dirección y velocidad en la escala intradiaria $(<24 \mathrm{~h})$, posiblemente debido al ciclo mareal, los efectos topográficos por la cercanía a la línea costera ( $1000 \mathrm{~m})$ y la presencia de variaciones de alta frecuencia del viento. Para los períodos de verano e invierno la Tabla 1 resume las direcciones predominantes de la corriente y sus intensidades medias asociadas. Durante los meses de febrero a marzo 1999 la dirección alterna en proporciones similares norte y este, aunque con una considerable número de casos que se revierten al sur. En el período de julio a septiembre 1999 la corriente sur tiende a predominar, aunque también existen muchos casos alternados. Las intensidades medias tienden a disminuir en el invierno, aunque la dirección norte contribuye con las mayores velocidades.

La descomposición vectorial de la corriente revela la alta varibilidad y reversiones de la dirección en varias escalas de tiempo, tanto en el eje norte como en el eje Este. La Fig. 3a muestra que ambas componentes varían muy asociadas en el período de verano 1999. Mientras que la Fig. 3b muestras que el diagrama de vector progresivo de la corriente resulta en un transporte neto hacia la playa en el sector la Rinconada. En la época de invierno, las componentes norte y este se muestran estrechamente asociadas y con menor variabilidad, aunque también con reversiones periódicas de la corriente (Fig. 4a). El diagrama de vector progresivo en este caso se observa con amplia variabilidad en los flujos y el transporte neto también resulta dirigido hacia la playa (Fig. 4b).

\section{Tabla 1}

Resúmen estadístico de corriente medidas en la bahía de Antofagasta en dos períodos; febrero-marzo (verano) de 1999 y julio-septiembre (invierno) de 1999. n es en número de vectores promedios horarios correspondientes a un total de 31 días y 75 días de verano e invierno respectivamente, \% corresponde la ocurrencia de cada dirección, e intensidad es la velocidad media para cada dirección en $\mathrm{cm} \mathrm{s}^{-1}$.

Summary statistics for current measurements in Bahía de Antofagasta during two periods; February/March (Summer) 1999 and July/September (Winter) 1999. $\mathrm{n}$ is the number of hourly averaging vectors, corresponding to $31 \mathrm{~d}$ and $75 \mathrm{~d}$ in Summer and Winter, respectively, \% is the percentage of occurrence of current directions, and Intensidad is the mean velocity for each direction $\left(\mathrm{cm} \mathrm{s}^{-1}\right)$.

\begin{tabular}{llllll}
\hline & & \multicolumn{5}{c}{ Febrero - marzo 1999 } \\
& Norte & Sur & Este & Oeste & Total \\
\hline $\mathrm{n}$ & 253 & 156 & 225 & 108 & 742 \\
$\%$ & 34 & 21 & 31 & 14 & 100 \\
Intensidad & 5.8 & 4.6 & 3.4 & 3.9 & 4.4 \\
\hline \multicolumn{5}{c}{ Julio - septiembre 1999 } \\
\hline $\mathrm{n}$ & 460 & 505 & 390 & 442 & 1797 \\
\% & 26 & 27 & 22 & 25 & 100 \\
Intensidad & 3.9 & 3.4 & 2.8 & 2.7 & 3.2 \\
\hline
\end{tabular}



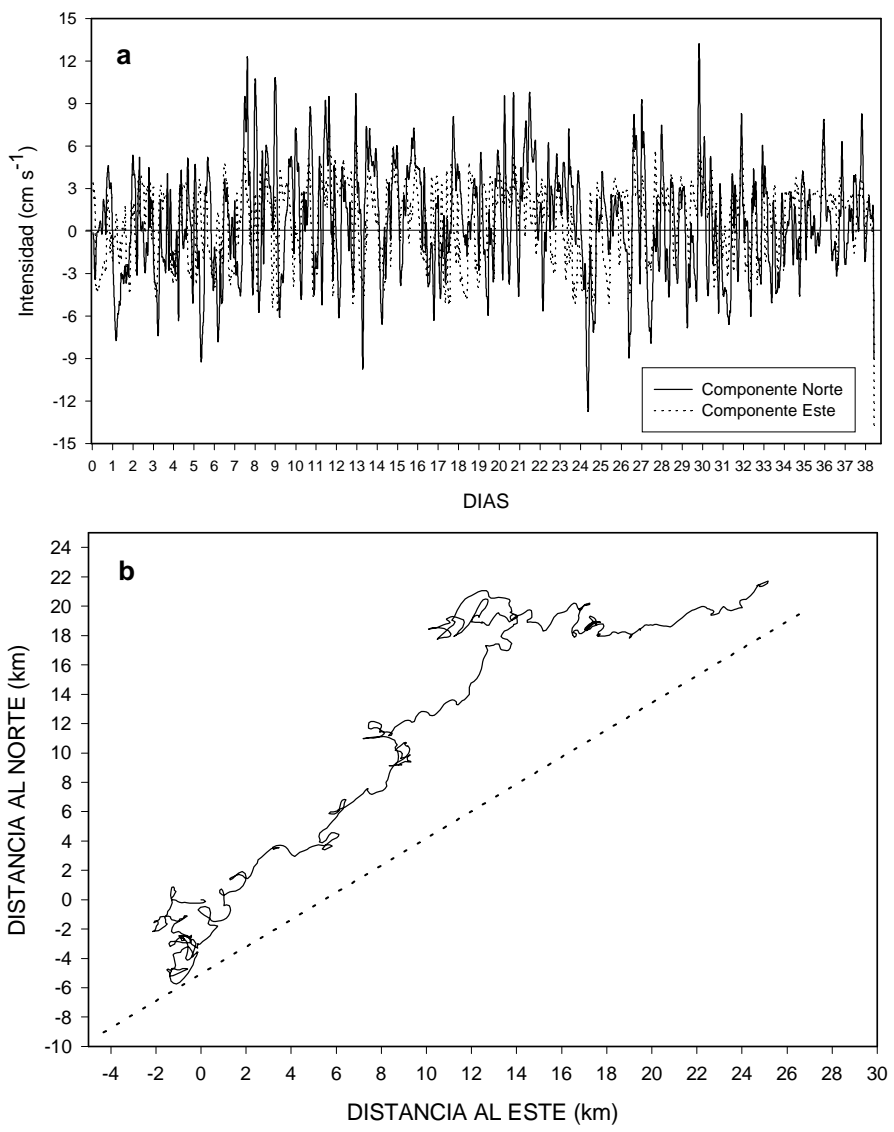

Figura 3

a) Componentes Norte y Este de la corriente en serie de tiempo (horas) para el período febrero a marzo de 1999. b) Vector progresivo de la corriente para el período febrero-marzo de 1999. La serie de corriente no ha sido filtrada para observar su variabilidad de alta frecuencia.

a) North and East components of currents (hourly vectors) for the period February/March 1999. b) Progressive vector of current for February/March 1999. The time series has not been filtered to fully capture its high frequency variation. baja contribución de la marea (Fig. 5b). Debe notarse que la correlación cruzada entre corriente y marea se realizó sin aplicación del filtro a la corriente para incluir el efecto marea de alta frecuencia $(<24$ h). Bajo las mismas consideraciones, la serie horaria de corrientes, vientos y marea de invierno, magnifican aún mas la fuerte contribución del viento a la varianza de corrientes. La Fig. 6a ilustra la asociación entre las componentes y la Fig. 6b confirma la fuerte correlación entre el viento y la corriente $(r>0.7)$, además de la baja contribución mareal ( $\mathrm{r}$ $<0.15$ ).

La presencia de una gran cantidad de componentes periódicos en las componentes de la corriente (Fig. 3 y Fig. 4) queda de manifiesto al aplicar una serie de Fourier a la componente norte de la corriente. La serie de Fourier permite analizar la varianza de la corriente en función de la frecuencia. De tal manera que se revelan los ciclos mayores que contribuyen a la varianza total de la serie. Para el período de verano, el pulso de varianza de mayor magnitud de la componente norte de la corriente y del viento se detectó en la ventana de 24 h. Se observó también un pulso importante a las $12 \mathrm{~h}$ y otro de 2 días. Tanto el de $24 \mathrm{~h}$ como de $12 \mathrm{~h}$ responden a ambas variables, corriente y vientos, no así el de 2 días (Fig. 7a). En el invierno el ciclo de $24 \mathrm{~h}$ presenta una alta densidad espectral para el viento y menor para la corriente, aunque aún se encuentran asociados. También se observó un ciclo de $5 \mathrm{~d}$ de la corriente asociado al viento (Fig. 7b).

Dada la importancia y prevalencia de los vientos sur en la zona y la estrecha asociación entre los ejes nortesur y este-oeste, con una mayor contribución del primero, se analizaron las variables forzantes de la corriente sobre la base del comportamiento de las componentes norte de la corriente y el viento. La contribución del viento y la marea a la variabilidad de las corrientes en la época de verano se ilustra en la Fig. 5. Las series filtradas (removido el componente mareal) de corrientes y vientos de verano varían asociadas en el tiempo, con una baja contribución de la marea a esta variabilidad (Fig. 5a). El análisis de correlación cruzada para estas series confirma la fuerte y significativa contribución del viento a la varianza de la corriente y la
El análisis de mediciones quincenales de la corriente en el sector La Rinconada sugiere cambios estacionales de las direcciones y velocidades dominantes en el sector La Rinconada. En verano, entre 0 y 50 m la dirección predominante observada es hacia el este y norte, con una reversión al sur y este en el otoño e invierno (Fig. 8a). Las mayores velocidades se concentran en fases dominantes al sur y con períodos de calma durante la transición estacional (Fig. 8b). La distribución vertical de la temperatura sugiere una conexión entre los cambios estacionales de la corriente y el levantamiento de las isotermas (Fig. 8c), posiblemente asociados a períodos de intensificación de la surgencia. 

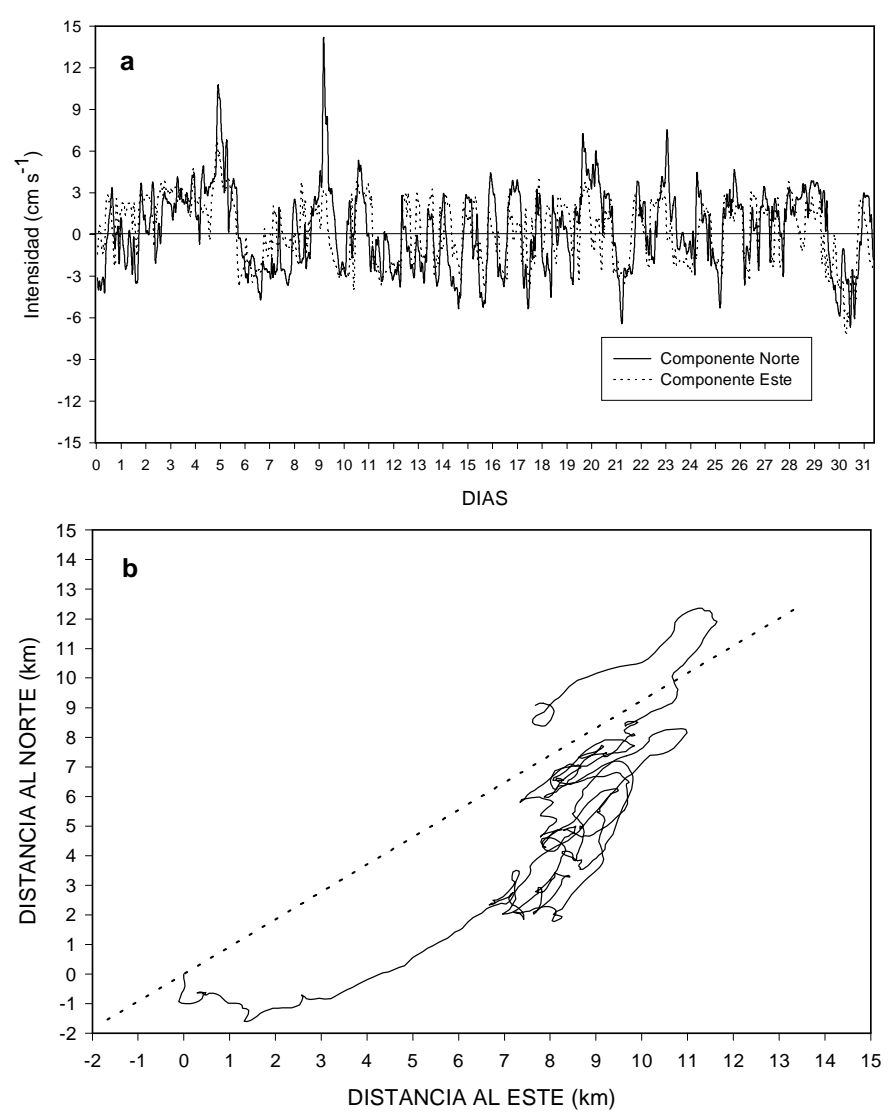

Figura 4

a) Componentes Norte y Este de la corriente en serie de tiempo (horas) para el período julio a septiembre de 1999. b) Vector progresivo de la corriente para el período julio-septiembre de 1999. La serie de corriente no ha sido filtrada para observar su variabilidad de alta frecuencia.

a) North and East components of currents (hourly vectors) for the period July/August 1999. b) Progressive vector of current for July/August 1999. The time series has not been filtered to fully capture its high frequency variation. indica que el flujo de agua tiende a ingresar por el sector noroeste de la bahía y a vaciarse a través del sector sureste. Adicionalmente, el campo de vectores de la Fig. 9 sugiere la presencia de un giro ciclónico (sentido horario) al interior de la bahía, el cual es consistente con la predominancia de la corriente hacia el noreste (hacia la costa) en el sector La Rinconada. Por otra parte la presencia de vectores tangenciales al eje entre ambas puntas sugiere una zona frontal en el sector sur de la boca de la bahía.

Desde la información del CTD, obtenida el mismo día de medición de corrientes directas (16 de agosto), se derivó la temperatura, densidad y oxígeno disuelto de la capa más superficial $(0-5 \mathrm{~m})$. La distribución de temperatura superficial del mar (TSM) durante el transcurso de medición de corrientes instantáneas muestra claramente la presencia de una cuña fría de agua que presiona desde el sector sur y suroeste a una masa de agua mas cálida al interior de la bahía (Fig. 10a). Esta zona frontal se manifiesta con mayor intensidad hacia el sector sur de la bahía de acuerdo a la distribución de densidad superficial (Fig. 10b). Es interesante observar que el cuerpo de agua fría contiene un menor grado de oxigenación (Fig. 10c) indicando que probablemente corresponde a aguas recientemente afloradas hacia el área sur externa a la bahía. El levantamiento de la oxiclina asociado a la surgencia es una característica de la zona (Morales et al. 1996).

Sobre el eje de la bahía, en el sentido surnorte, en una sección transversal la distribución vertical de la temperatura revela la presencia del frente en el extremo sur que

\section{Variación espacial}

Durante el mes de agosto de 1999 la medición directa de corrientes sobre el eje que une las dos puntas de la bahía se realizó el día 16 en condiciones de relativa calma, con condiciones de viento sur de intensidades débiles $\left(1\right.$ a $\left.4 \mathrm{~m} \mathrm{~s}^{-1}\right)$. La transecta se completó en un período de $3 \mathrm{~h}$ durante la fase llenante de marea. Las velocidades de corrientes fluctuaron con una media de $30 \mathrm{~cm} \mathrm{~s}^{-1}$. La velocidad media en la capa superficial $(0-10 \mathrm{~m})$ fue de $31.8 \mathrm{~cm} \mathrm{~s}^{-1}$, en la capa media $(10-50 \mathrm{~m}) 28.9 \mathrm{~cm} \mathrm{~s}^{-1}$, mientras que en la capa profunda $(50-100 \mathrm{~m})$ la velocidad media fue de $27.7 \mathrm{~cm} \mathrm{~s}^{-1}$. En cada punto de medición, con excepción en la zona de Punta Coloso, la dirección fue muy similar en la tres capas. La Fig. 9 presiona el cuerpo de agua más interno induciendo un levantamientos de las isotermas a la entrada sur de la bahía (Fig. 11a). La distribución vertical de salinidad muestra el frente menos salino en el sector sur, y adicionalmente revela la presencia de anillos de menor salinidad en la capa superior de $50 \mathrm{~m}$, que pueden reflejar cuerpos de aguas retenidos en la bahía (Fig. 11b). Finalmente el frente en el extremo sur se muestra en el levantamiento de la oxiclina, con la presencia de aguas pobres en oxígeno $\left(<5 \mathrm{ml} \mathrm{L}^{-1}\right)$ al interior de la capa de $10 \mathrm{~m}$ superiores (Fig. 11c). De acuerdo a la Fig.11, es importante notar que la influencia del frente se manifiesta fundamentalmente en el estrato superior de los $40 \mathrm{~m}$, sugiriendo que el potencial desplazamiento del frente en el eje transversal a la bahía debido a 
fluctuaciones en intensidad del foco de surgencia,

más superficial. incidirá mayoritariamente en la circulación de la capa
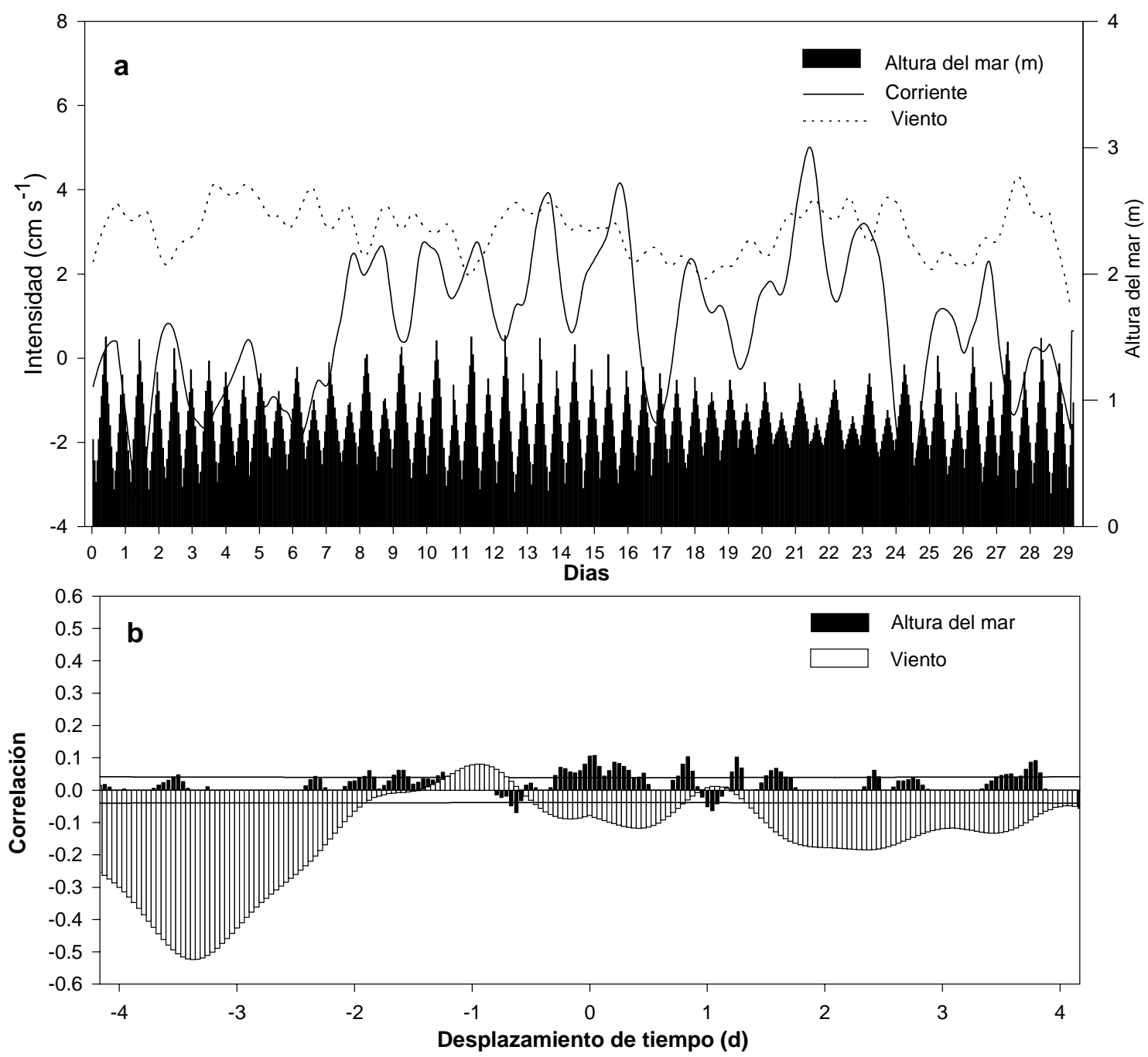

Figura 5

a) Componentes en el eje norte-sur de la corriente y el viento y la altura horaria del mar durante febrero-marzo de 1999 en la bahía de Antofagasta. Las componentes de la corriente y el viento se filtraron mediante filtro pasa-bajo para remover variaciones de alta frecuencia $(<24$ h). b) Función de correlación cruzada entre la componente norte de la corriente, el viento y la marea. La líneas contínuas ilustran el valor crítico a una probabilidad de 0.05 .

a) North components of current and wind, and hourly sea height during February/March 1999 in Bahía de Antofagasta. The components of current and wind were filtered by the lowess filter to remove high frequency $(<24 \mathrm{~h})$ variation. b) Cross-correlation functions between current, wind and sea height. Continuous lines indicate critical values $(\mathrm{p}<0.05)$. 

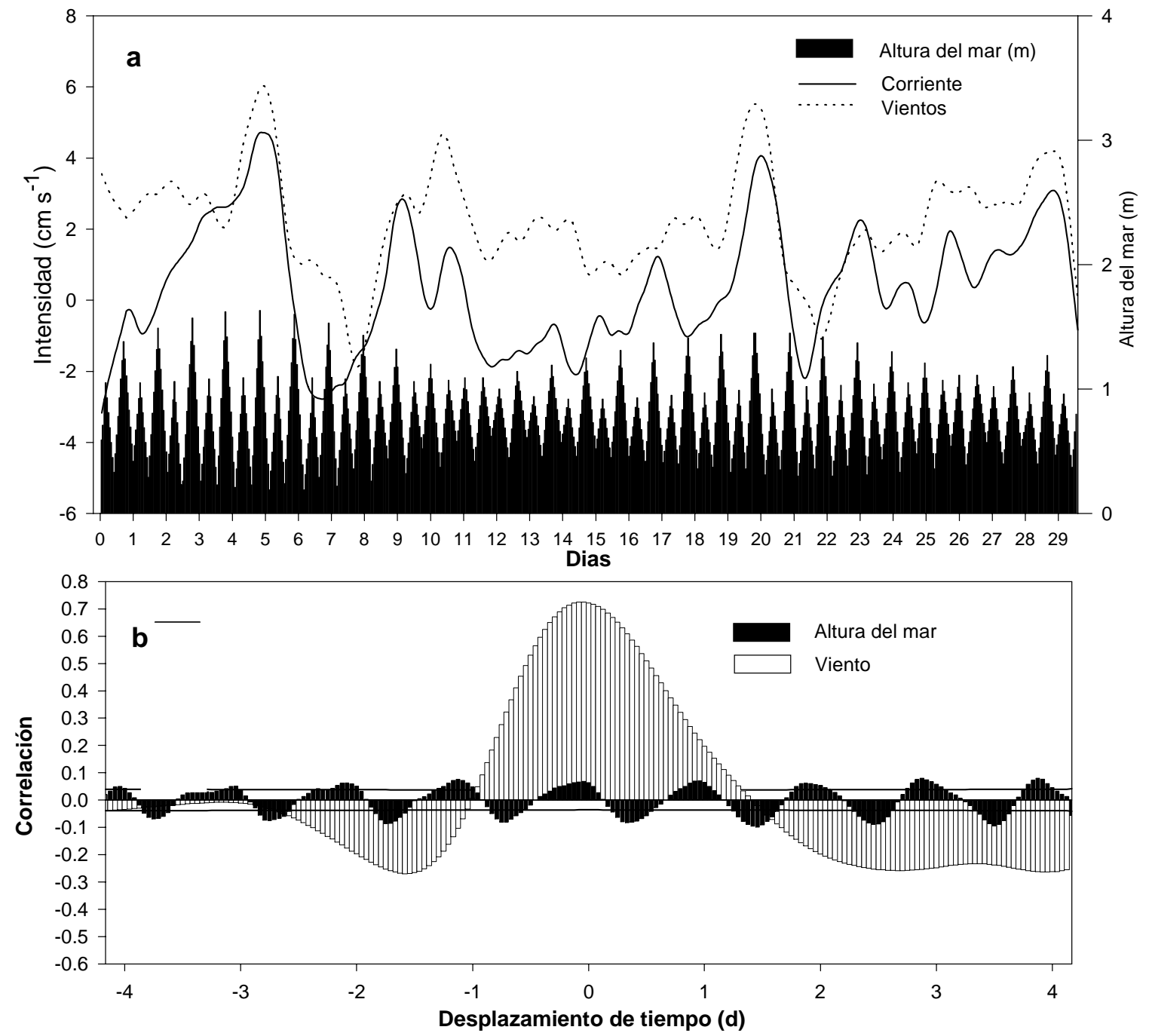

Figura 6

a) Componentes en el eje norte-sur de la corriente y el viento y la altura horaria del mar durante julio-agosto de 1999 en la bahía de Antofagasta. Las componentes de la corriente y el viento se filtraron mediante filtro pasa-bajo para remover variaciones de alta frecuencia ( $<24$ h). b) Función de correlación cruzada entre la componente norte de la corriente, el viento y la marea. La líneas contínuas ilustran el valor crítico a una probabilidad de 0.05 .

a) North components of current and wind, and hourly sea level during July/August 1999 in Bahía de Antofagasta. The components of current and wind were filtered by the lowess filter to remove high frequency $(<24 \mathrm{~h})$ variation. b) Cross-correlation functions between current, wind and sea level. Continuous lines indicate critical values $(\mathrm{p}<0.05)$. 


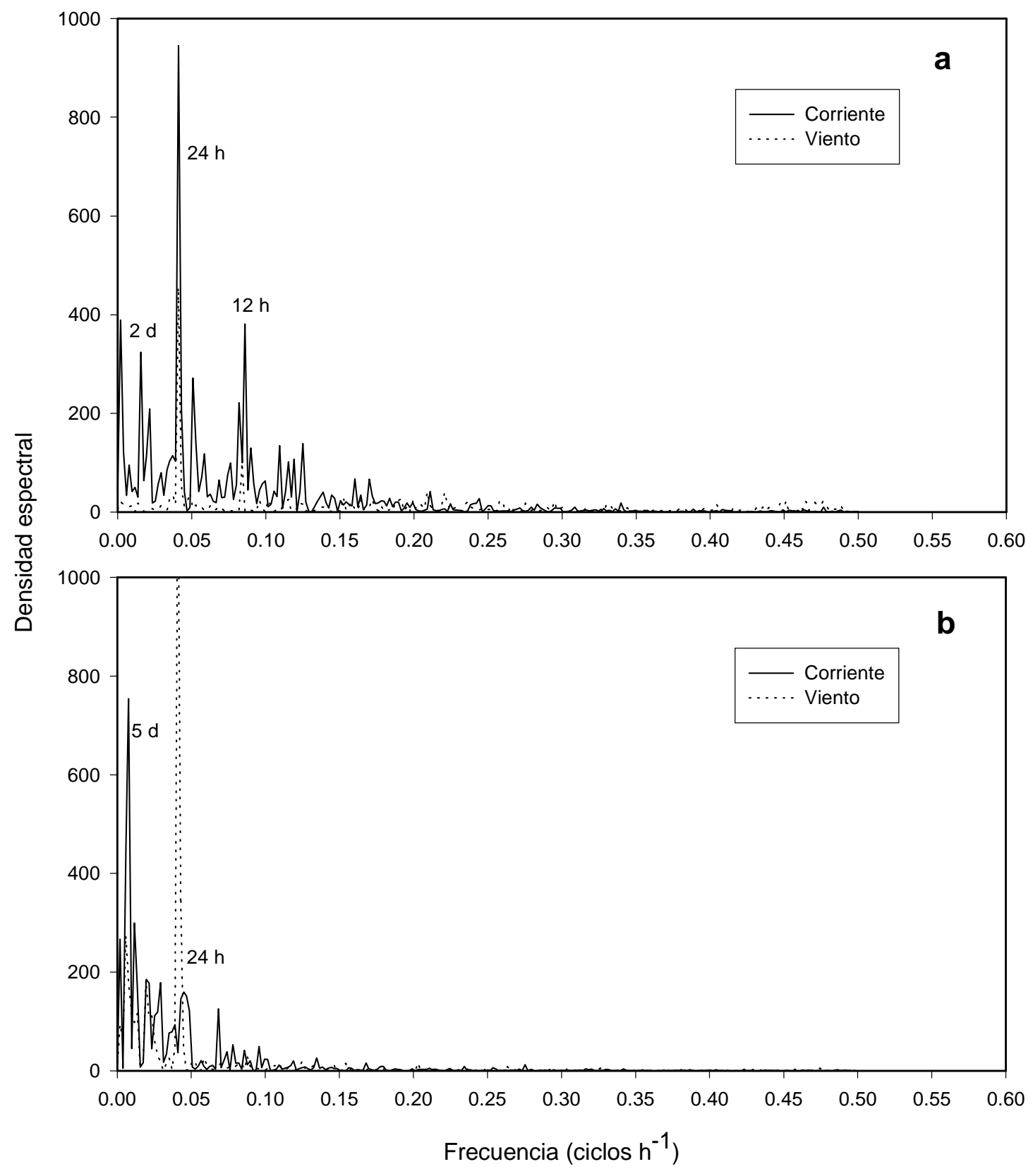

Figura 7

Análisis espectral de la componente Norte de la corriente y del viento mediante series de Fourier para el verano (a) y el invierno (b) de 1999 en bahía de Antofagasta.

Power spectra of north components of current and wind after Fourier analysis for Summer (a) and Winter (b) 1999 in Bahía de Antofagasta. 


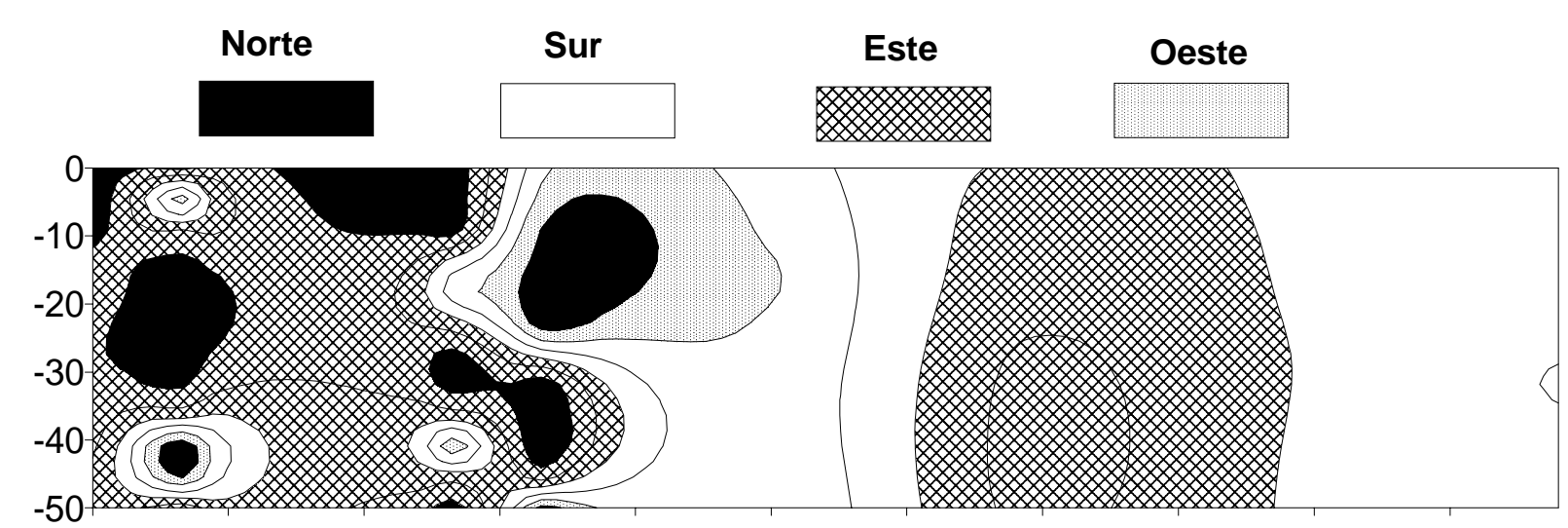

a

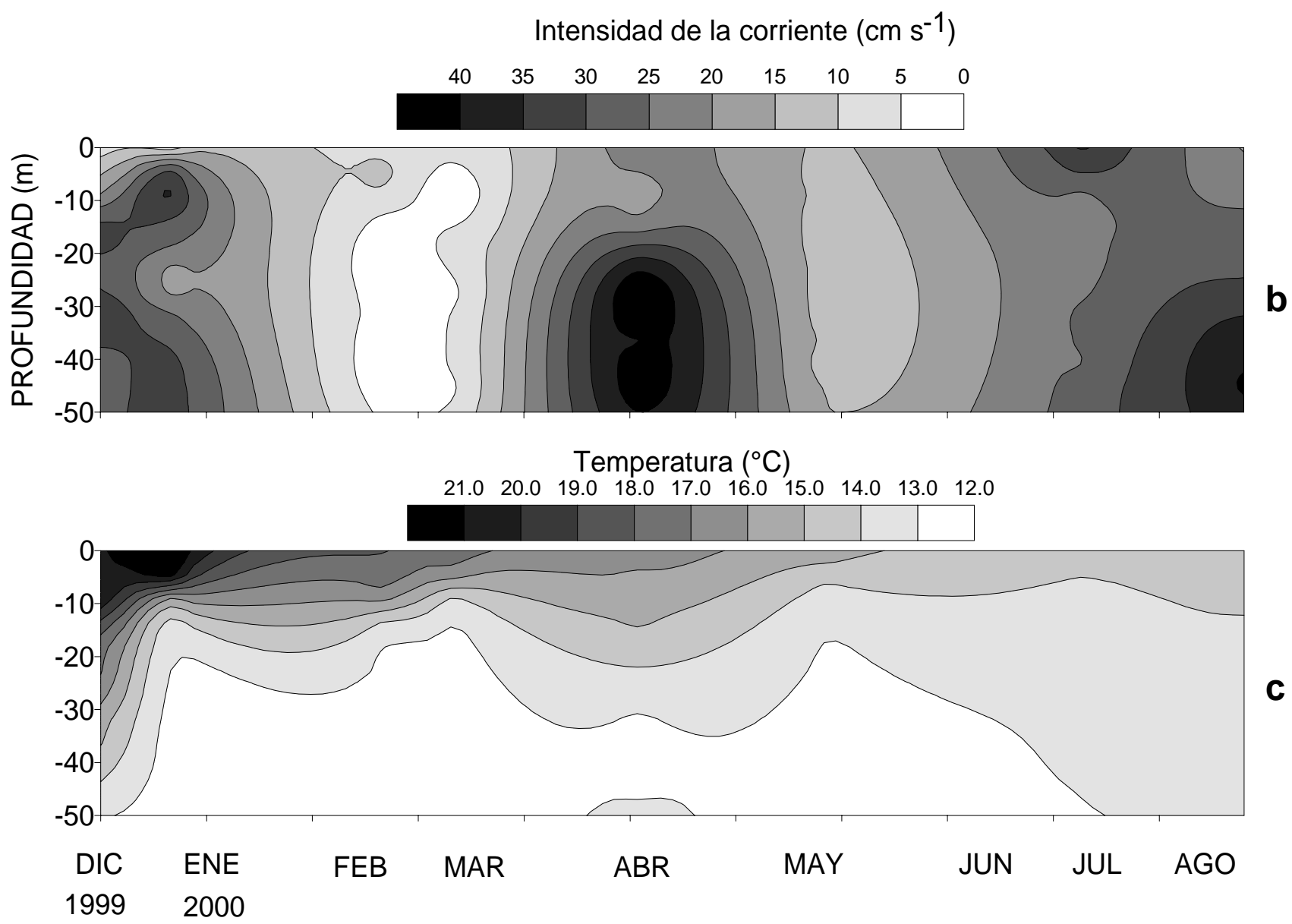

Figura 8

Variación estacional de la corriente y temperatura en bahía de Antofagasta 1999-2000, desde serie quincenal de medición de perfiles de corrientes instantáneas y temperatura. Dirección (a) y velocidad (b) de la corriente y de la temperatura (c) en la capa de 50 m durante desde diciembre de 1999 a agosto de 2000.

Seasonal variation of currents and temperature in Bahía de Antofagasta 1999-2000, from a time series (15 d intervals) of current profiling and temperature. Direction (a) and velocity (b) of current and temperature (c) in the upper de 50 m during December 1999 trough August 2000. 


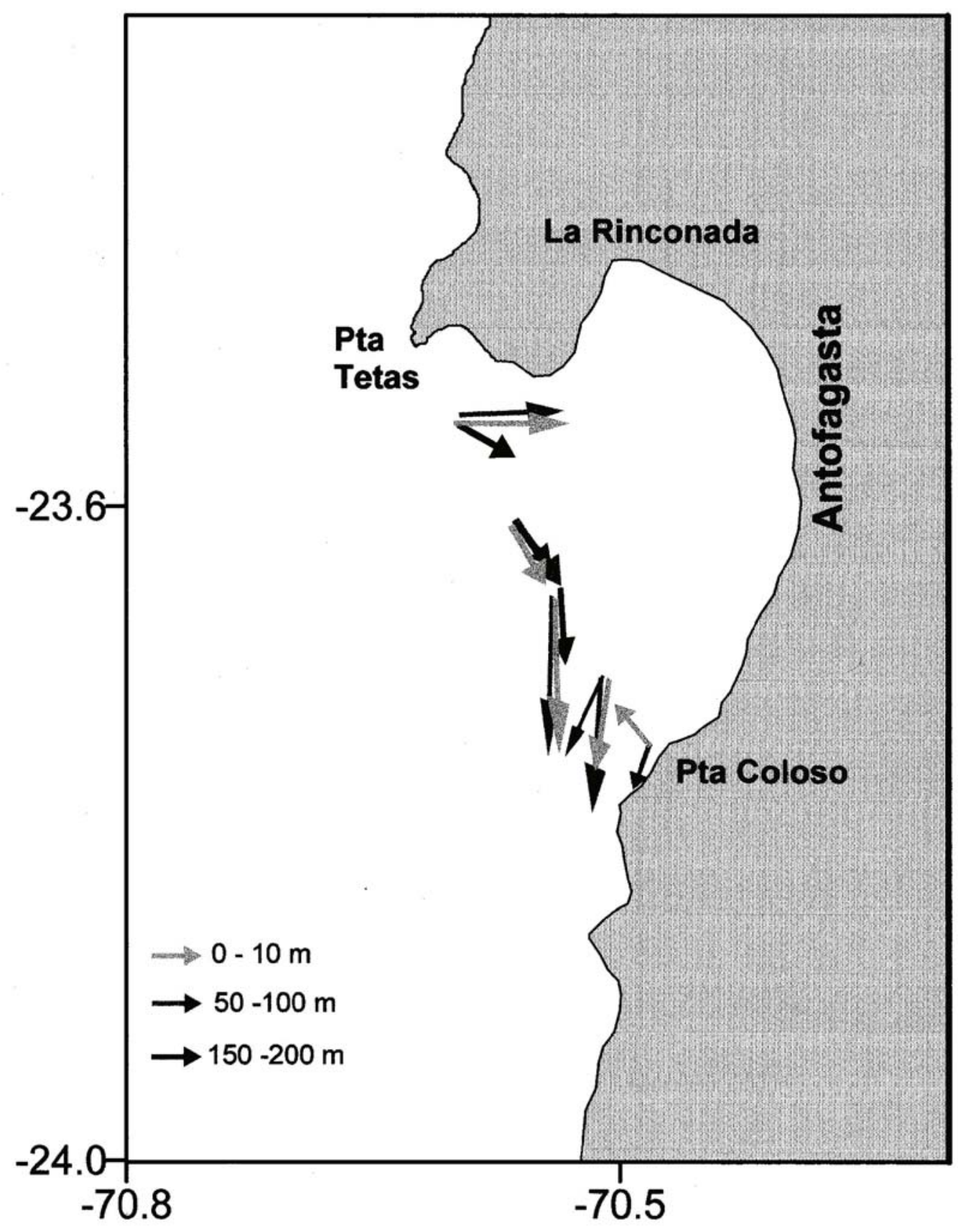

Figura 9

Distribución horizontal de la corriente en 3 capas sobre el eje de entrada de bahía Antofagasta, el día 16 de agosto de 1999 , obtenida desde perfiles de corriente instantánea mediante sensor Doppler. La magnitud de los vectores fluctuó entre 20 - $40 \mathrm{~cm} \mathrm{~s}^{-1}$.

Instantaneous currents in 3 layers over the entrance axis of Bahía de Antofagasta, on August 16, 1999, obtained from vertical profiles of currents using a Doppler sensor. The magnitude of the vectors was between $20-40 \mathrm{~cm} \mathrm{~s}^{-1}$. 

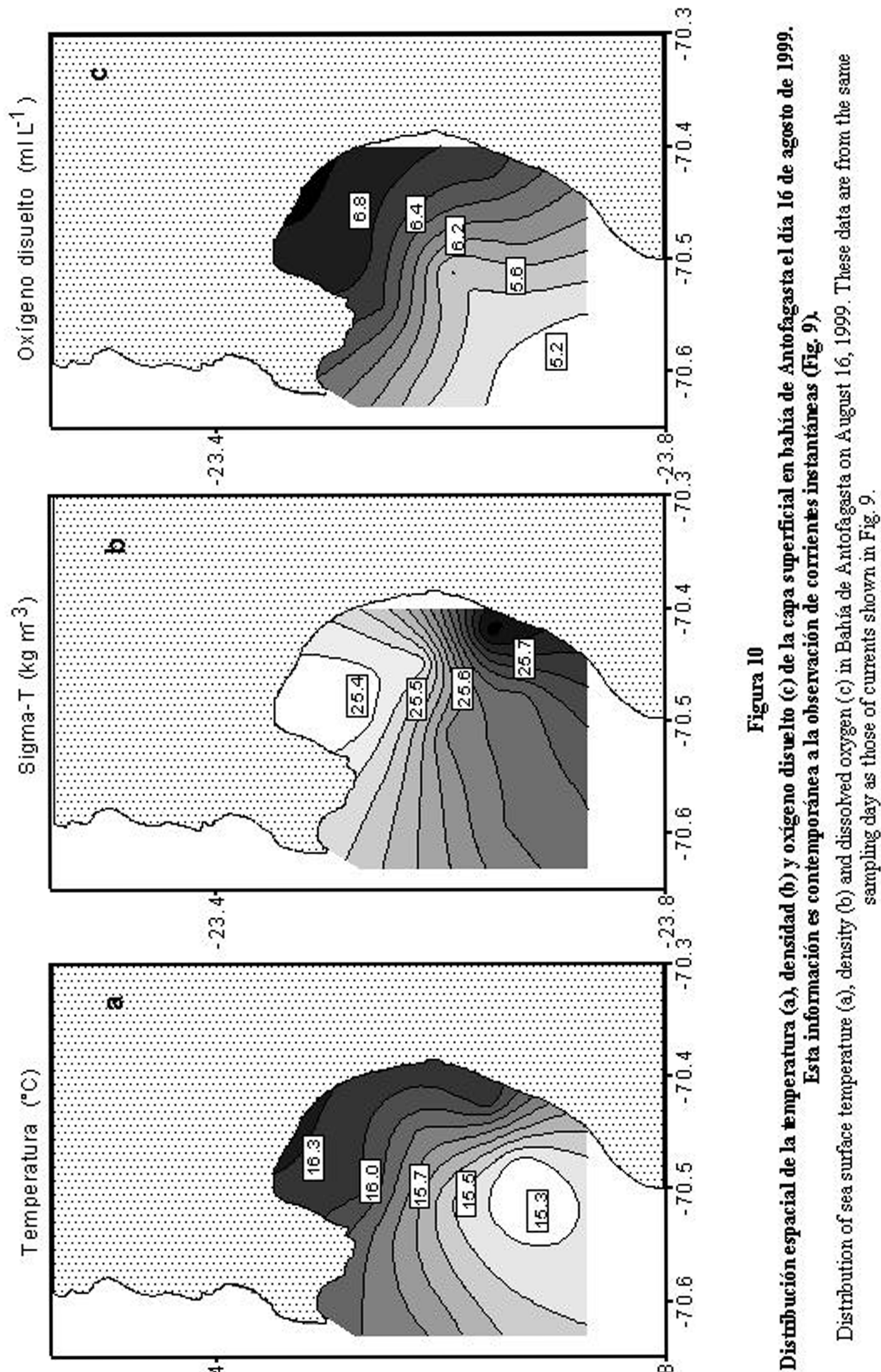

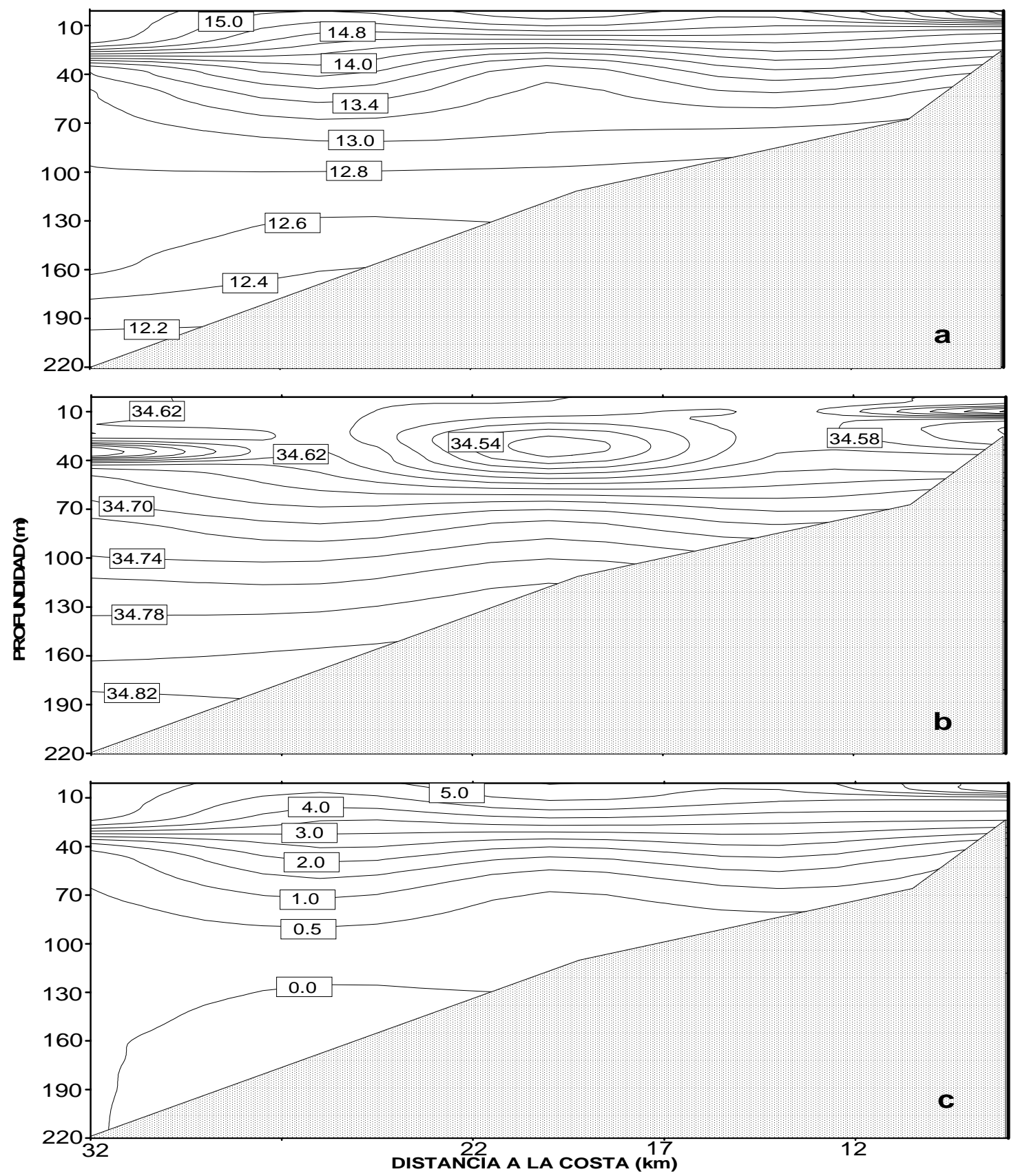

Figura 11

Secciones transversales de condiciones oceanográficas de Bahía Antofagasta, el día 16 de agosto de 1999, en el eje latitudinal (Fig. 2), mostrando la distribución vertical de temperatura (a), salinidad (b) y oxígeno disuelto (c).

Oceanographic sections of Bahía de Antofagasta, on August 16, 1999, over the latitudinal axis (Fig. 2), illustrating the vertical distribution of temperature (a), salinity (b) and dissolved oxygen (c). 


\section{Discusión}

La información proveniente de las series de tiempo en el sector La Rinconada, que representa un punto receptor final del cuerpo de agua que ingresa a la bahía, revela variabilidad de alta frecuencia, dada su cercanía a la playa $(\sim 1000 \mathrm{~m})$ y baja profundidad $(<50 \mathrm{~m})$. La incidencia de las fases de la marea y varianza introducida por la morfología de la costa y posiblemente la topografía del fondo se refleja en los cambios en escalas de horas que ocurren en las componente de la corriente (Fig. 3 y Fig. 4). De hecho, la presencia de ciclos periódicos asociados a la marea, 6 y 12 h, se detectaron en el análisis espectral (Fig. 7). No obstante, el efecto marea es débil según nuestros análisis y es relevante observar que los ciclos que mayor varianza explican (>24 h) se asocian fuertemente al viento local. Además, el flujo neto de transporte hacia la costa es muy consistente con la observación espacial de la corriente. De manera tal que el sitio de muestreo, a pesar de su variabilidad de escala menor, puede reflejar la dinámica mas global de circulación de la bahía.

El análisis integrado de la información, removido el efecto mareal, sugiere que el forzante fundamental de la circulación la constituye la surgencia activa al sur de la bahía. Los estudios previos (Fonseca \& Farías 1987, Escribano \& McLaren 1999, Escribano et al. 2000a) muestran la persistencia de este sitio de surgencia a través del año, localizado al sur de Antofagasta y que genera una pluma de surgencia de orientación norte o noroeste. La alta variabilidad en la intensidad de vientos sur y suroeste, que se manifiestan en escalas diarias y en horas en la zona a través del año (Rutllant 1993, Rutllant et al. 1998), también observada en nuestros datos, sugiere la ausencia de un efecto uniforme del foco de surgencia, sino mas bien que tal influencia sería pulsátil y altamente variable día a día. Tal situación da lugar a una zona frontal en la boca sur de la bahía, que se manifestó en nuestro datos y que puede fluctuar en posición. No obstante su presencia mantiene un sistema de alta capacidad de retención del agua mas interna de la bahía. El efecto forzante de la cuña de agua fría sobre el cuerpo de agua más cálido de la bahía, puede a su vez inducir una reversión del flujo de agua hacia el sur. El mecanismo de acción del foco de surgencia puede modelarse conceptualmente en tres fases:

I. Intensificación de la componente sur del viento, induciendo surgencia activa al sur de la bahía y pulsando la pluma fría hacia el norte, llenando la bahía.

II. Relajación de la surgencia y reversión del flujo hacia el sur, favorecido por la morfología de la costa y topografía.
III. Formación de la zona frontal en el sector sur de entrada de la bahía retardando el flujo al sur y favoreciendo una circulación ciclónica al interior de la bahía.

La Fig. 12 ilustra gráficamente el modelo de circulación en tres fases. Es interesante observar que la dinámica de circulación se puede explicar sobre la base de una capa de circulación. La circulación clásica de Ekman se ha descrito usualmente en dos flujos compensatorios, la capa superficial de Ekman y un flujo compensatorio subsuperficial (Pickard \& Emery 1990, Smith 1995). Es muy probable que la topografía de fondo de la bahía y su morfología costera son forzantes importantes para determinar la ausencia de un flujo compensatorio en la capa profunda. No obstante, la surgencia costera en la zona parece romper el esquema clásico de Ekman, dado un efecto barotrópico de una corriente norte predominante en el sector qu inhibe el flujo compensatorio a la capa de Ekman (Marín et al. en prensa). Este flujo mantiene una corriente residual ( 10 $\mathrm{cm} \mathrm{s}^{-1}$ ) en la zona de Antofagasta (Marín et al. en prensa).

En sistemas de surgencia de otras zonas, se han descrito las zonas frontales que encierran aguas costeras, favoreciendo áreas de retención. La masa de agua retenida se le ha nominado como "sombra de surgencia" (Graham et al. 1992). Estas áreas actuan como un eficiente mecanismo para retener agregaciones planctónicas (Graham \& Largier 1997). Bahía de Antofagasta parece constituir un sistema semi-cerrado sujeto a una sombra de surgencia. De esta manera es posible explicar las densas agregaciones de organismos en su interior (Escribano \& Hidalgo 2000). Sin embargo, en adición a la supuesta sombra de surgencia, la presencia de un giro ciclónico en su interior, genera un sistema aún más eficiente para la retención de la masa de agua. Tales giros son determinantes como mecanismos que regulan los tiempos de residencia de poblaciones planctónicas en áreas costeras (Wrobleswki 1982, Roy 1998) y que influencian la dinámica de reclutamiento de organismos bentónicos, a través de la retención de fases meroplanctónicas (Wing et al. 1998). Es importante señalar, sin embargo, que si se considera la alta variabilidad en los pulsos de surgencia, inducida por la fluctuaciones de alta frecuencia de los vientos, la renovación de la masa de agua interna puede ocurrir en forma pulsátil a través del extremo sur de la bahía. Este sector actua como una zona de transición y mezcla entre las aguas internas y externas de la bahía, posibilitando el intercambio de las aguas y sus propiedades. No obstante, se debe notar que potenciales reversiones de la corriente en ese sector, producto de la variabilidad del foco de surgencia, serán manifiestas en la capa superior ( $<40 \mathrm{~m})$, mientras que el estrato profundo debería ser mas estable y con flujo mayoritario hacia el sur, 
vaciando la bahía. Además la variación en la localización del frente de surgencia puede, de acuerdo al efecto topográfico (Wolanski \& Hammer, 1988), inducir variación latitudinal en la interacción flujo/reflujo de aguas y sus propiedades en el sector sur frente a Punta Coloso.

\section{Agradecimientos}

Este estudio ha sido financiado por Minera Escondida Ltda. Estamos agradecidos a Andrés Caamaño quien facilitó información de corrientes y vientos del sector de Coloso. También agradecemos a Julio Duarte de la Dirección General de Aeronáutica quien gentilmente nos facilitó información de vientos del aeropuerto Cerro Moreno. Varios estudiantes han colaborado en los diferentes muestreos, entre ellos Manuel Rojo, Diego Fernández y Luis Figueroa. Estamos agradecidos de dos revisores anónimos, quienes contribuyeron sustancialmente a mejorar el trabajo y sus interpretaciones.

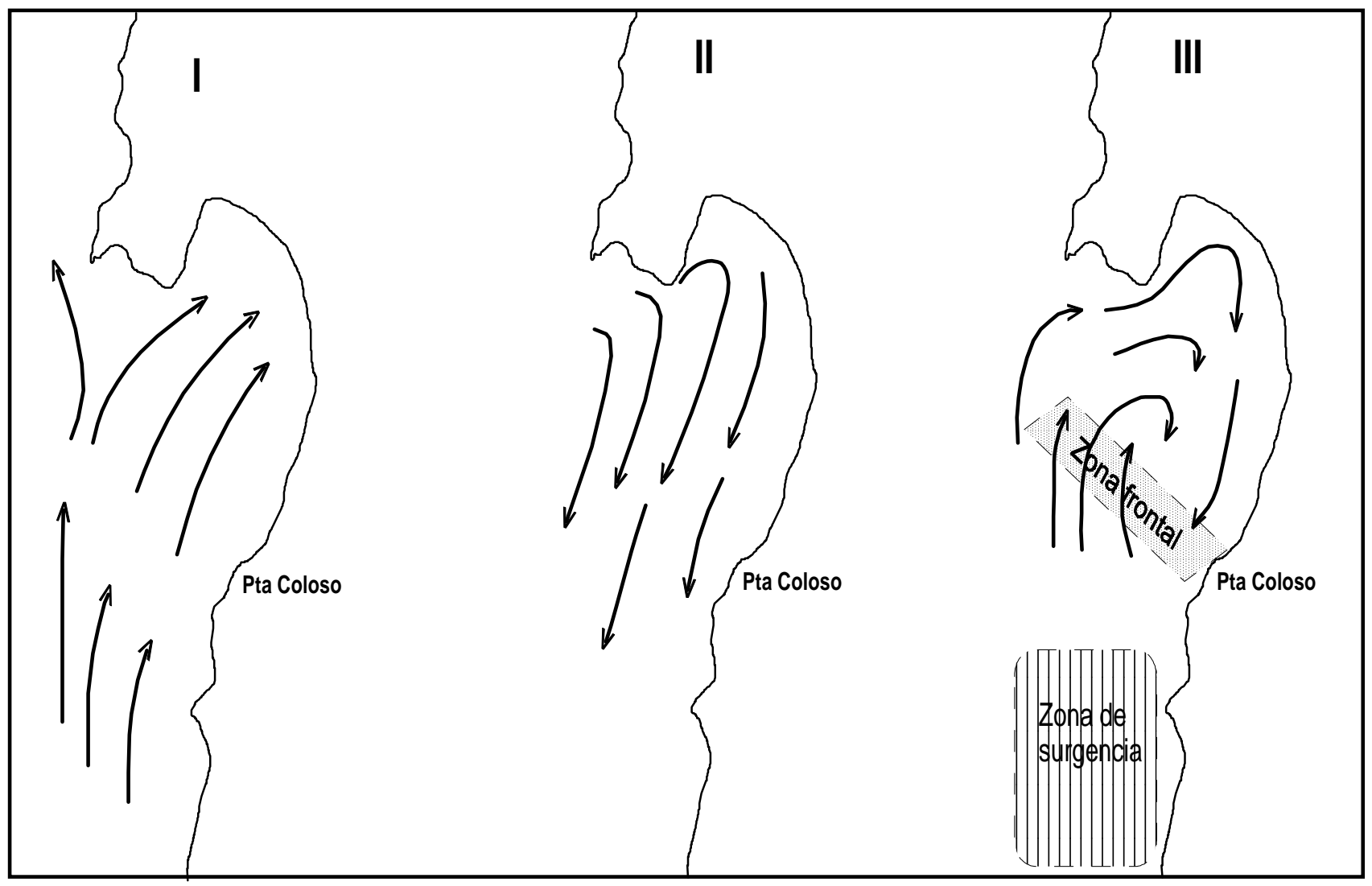

Figura 12

Modelo conceptual de circulación forzada por el viento y la surgencia de bahía de Antofagasta. El modelo se manifiesta en 3 fases dinámicas: I: fase inicial forzante del viento que induce surgencia y llenado de la Bahía desde el Sur, II: fase intermedia de relajación de vientos y reflujo del cuerpo de agua, III: desarrollo de zona frontal induciendo zona de retención interior y giro ciclónico de la corriente.

Conceptual model of wind-driven circulation coupled to upwelling in Bahía de Antofagasta. The model illustrates 3 dynamical phases:

I: initial stage forced by upwelling-inducing wind causing water inflow to the bay from the South, II: intermediate stage of wind relaxation and flow reversing of the water body, III: development of an upwelling front inducing the retention zone and cyclonic eddy inside the bay. 


\section{Literatura Citada}

Alheit $\mathbf{J}$ \& $\mathbf{P}$ Bernal. 1983. Effects of physical and biological changes on the biomass yield of the Humboldt Current Ecosystem, In: Large Marine ecosystems. V.: Stress, mitigation and sustainability. Sherman, K., Alexander, L.M. \& B.D. Gold (eds). American Association for the Advancement of Science, Washington D.C., pp-53-68. pp.

Barber RT \& RL Smith. 1981. Coastal upwelling ecosystems. In Longhurst, A.R. (ed). Analysis of Marine Ecosystem. Academic Press, pp. 31-68.

Beer T. 1989. Applied environmetrics oceanographic tables. Applied Evironmentrics, Victoria Australia.38 pp.

Box GEP \& GM Jenkins. 1976. Time Series Analysis: Forecasting and Control. Revised Edition. Oakland, CA: Holden-Day Inc. 384 pp.

Escribano R \& IA McLaren. 1999. Production of Calanus chilensis in the upwelling area of Antofagasta, northern Chile. Marine Ecology Progress Series 177: 147-156.

Escribano R \& $\mathbf{P}$ Hidalgo. 2000. Spatial distribution of copepods in the north of the Humboldt Current region off Chile during coastal upwelling. Journal of the Marine Biological Association U.K. 80: 283-290.

Escribano R, Marin V, Hidalgo P \& G Olivares. 2000a. Physical-biological interactions in the nearshore zone of the northern Humboldt Current ecosystem. International Symposium, Bahías y Costas Abiertas: conecciones. PUC, Octubre 2000, Santiago, Chile.

Escribano, R, Marín V \& C Irribarren. 2000b. Distribution of Euphausia mucronata at the upwelling area of Peninsula Mejillones: the influence of the oxygen minimum layer. Scientia Marina. 64: 69-77.

Fonseca T. 1989. An overview of the Poleward Undercurrent and upwelling along the Chilean coast. In Poleward flows along Eastern Ocean Boundaries, S. J. Neshyba, C.N.K. Mooers, R. L. Smith and R. T. Barber (eds). Spring-Verlag, New York, pp. 203-228.

Fonseca TR \& M Farías. 1987. Estudio del proceso de surgencia en la costa chilena utilizando percepción remota. Investigaciones Pesqueras 34:33-46.

Graham W \& JL Largier. 1997. Upwelling shadows as nearshore retention sites: the example of northern Monterey Bay. Cont. Shelf Res. 17:509-532.

Graham W, J Field \& D Potts. 1992. Persistent “upwelling shadows" and their influence on zooplankton distributions. Mar. Biol. 114: 561-570.

Hutchings L, GC Pitcher, TA Probyn \& GW Bailey. 1995. The chemical and biological consequences of coastal upwelling. In C.P. Summerhayes, K.-C. Emeis, M.V. In. Angel, R.L. Smith \& B. Zeitzschel (eds), Upwelling in the Ocean: Modern Processes and Ancient Records. John Wiley \& Sons, New York. pp. 65-82.
Marín VH, Escribano R, Delgado LE, Olivares G \& P Hidalgo. (en prensa). Upwelling in a coastal site off the northern Humboldt Current System: spatial structure and biological consequences. Continental Shelf Research.

Morales CE, JS Blanco, M Braun, $\mathbf{H}$ Reyes \& N Silva. 1996. Chlorophyll- $a$ distribution and associated oceanographic conditions in the upwelling region off northern Chile during the winter and spring 1993. DeepSea Res. 43: 267-289.

Peterson W. 1998. Life cycle strategies of copepods in coastal upwelling zones. Journal of Marine Systems 15: 313-326.

Pickard GL \& WJ Emery. 1990. Descriptive physical oceanography. Pergamon Press. Toronto, Canada.

Robles F, E Alarcon \& A Ulloa. 1976. Las masas de agua en la región norte de Chile y sus variaciones estacionales en un período frío (1967) y en períodos cálidos (1969, 19711973). Reunión de Trabajo sobre el fenómeno conocido como “El Niño”. Guayaquil, Ecuador, 4-12 de Diciembre de $1974, N^{\circ} 185: 94-196$.

Rodriguez L, Escribano R, Grone G, Irribarren C \& H Castro. 1996. Ecología del fitoplancton en la bahía de Antofagasta (23 S), Chile. Revista de Biología Marina, Valparaíso 31: 1-16

Roy C. 1998. An upwelling-induced retention area off Senegal: A mechanism to link upwelling and retention processes. In: Benguela Dynamics: Impacts of Variability on Shelf Sea Environments and their Living Resourses. Pillar, S., C. Moloney, A. Payne \& F. Shillington (Eds.) South African Journal of Marine Sciences 19: 89-98.

Rutllant J. 1993. Coastal lows and associated southerly winds events in north-central Chile. Preprints IV International Conference on Meteorology and Oceanography of the southern hemisphere. Hobart, Australia, March-April 1993. American Meteorological Society pp- 268-269

Rutllant J, H Fuenzalida, R Torres \& D Figueroa. 1998. Interacción océano-atmósfera-tierra en la región de Antofagasta (Chile $23^{\circ} \mathrm{S}$ ). Experimento Diclima. Revista Chilena Historia Natural 71: 405-427.

Silva SN \& HA Sievers. 1981. Masas de agua y circulación en la región de la rama costera de la Corriente de Humboldt: latitudes $18^{\circ} \mathrm{S}-33^{\circ} \mathrm{S}$ (operación oceanográfica MARCHILE X - ERFEN I). Ciencia y Tecnología del Mar. CONA 5: 5-50.

Smith RL. 1995. The physical processes of coastal ocean upwelling systems. En: Upwelling in the ocean: modern processes and ancient records. Summerhayes, C. P.; K. C. Emeis, M. V. Angel, R. L. Smith and B. Zeitzchel (eds). Pp. 39-64. John Wiley \& Sons. 
Strub P, Mesias J, Montecino V \& J Rutlland. 1998. Coastal ocean circulation off western South america. In: Robinson, A., Brink, K. (eds.), The Sea, Vol.2. The Global coastal ocean. First Workshop on Coastal Ocean Advanced Science and Technology Studies (COASTS) IOC, Liege. John Wiley \& Sons, Inc.

Walsh JJ. 1991. Importance of continental margins in the marine biogeochemical cycling of carbon and nitrogen. Nature 350: 53-55.

Wing S, L Botsford, S Ralston \& J Largier. 1998. Meroplanktonic distribution and circulation in a coastal retention zone of the northern California upwelling system. Limnology \& Oceanography 43: 1710-1721.
Wolanski E \& W Hamner. 1988. Topographically controlled fronts in the ocean and their biological influence. Science 241: 177-181.

Wroblewski JS. 1982. Interaction of currents and vertical migration in maintaining Calanus marshallae in the Oregon upwelling zone - a simulation. Deep Sea Research 29: 665-686. 